\title{
Buscando nuevas formas de expresión y relación para las personas con parálisis cerebral en proceso de envejecimiento a través de las TIC
}

\author{
Montserrat Santamaría Vázquez \\ Departamento Ciencias de la Educación. Universidad de Burgos, España \\ msvazquez@ubu.es \\ Yolanda González Alonso \\ Departamento Ciencias de la Educación. Universidad de Burgos, España \\ Concepción Manso Román \\ Centro de Atención Integral Fuentecillas. Asociación de Parálisis Cerebral, APACE. Burgos, España \\ Roberto Pérez Porras \\ Centro de Atención Integral Fuentecillas. Asociación de Parálisis Cerebral, APACE. Burgos, España
}

\begin{abstract}
Cerebral palsy (CP) describes a group of permanent disorders of the development of movement and posture, causing activity limitation, that are attributed to non progressive disturbances that occurred in the developing fetal or infant brain. The increasing life expectancy in people with CP allows speaking about people with $\mathrm{CP}$ in aging process from 45 years old. The CP Association of Burgos carried out an education proposal which includes an Information and Comunication Technologies (ICTs) workshop. The aim of the proposal was to increase their social relations and create new forms of expression using ICTs. They have learned how to use Windows, word processor programs, how to manage open softwaresand Internet access and they have developed searching strategies, managed e-mail and participated in social networks. The experience has increased their self-esteem and their sense of achievement. These feelings allow to break the stereotype of elderly people and disability.
\end{abstract}

Key words: Aging, Cerebral Palsy, ICTs.

\section{Resumen}

La parálisis cerebral (PC) es un grupo de trastornos permanentes del desarrollo, del movimiento y de la postura, que causan limitaciones en la actividad y que son atribuidos a alteraciones no progresivas ocurridas en el desarrollo cerebral del feto o del lactante. La esperanza de vida se ha incrementando en las personas con PC y, a partir de los 45 años, podemos hablar de personas en proceso de envejecimiento. Con el objetivo de fomentar las relaciones sociales y generar nuevas formas de expresión utilizando las tecnologías de la información y la comunicación (TIC), la Asociación de Personas con PC de Burgos realizó una propuesta educativa que incluía un taller de introducción y manejo del uso de las Tic. Se les entrenó en el acceso al entorno Windows, el manejo de procesador de texto, el uso de diferentes softwares libres, el acceso a Internet y el desarrollo de estrategias de búsqueda de información y conocimiento, el manejo del correo electrónico y la participación en redes sociales. La experiencia supuso un aumento del sentimiento de logro y de la autoestima, facilitando así romper con los estereotipos de persona mayor y discapacidad.

Palabras clave: envejecimiento, parálisis cerebral, TIC. 


\section{Introducción}

Las tecnologías de la información y la comunicación (TIC) están experimentando avances considerables en los últimos tiempos; sin embargo, son muchos los ámbitos en los que encontramos que estas tecnologías no están implantadas, provocando una brecha de exclusión derivada. Especialmente esta brecha de exclusión la sufren las personas que presentan limitaciones funcionales, ya sea por discapacidad o por motivos de edad, y en general todas las personas que por una u otra razón tienen necesidades diferentes a las consideradas normales (Ochoa, 2010).

El plan de acción Envejecer mejor en la sociedad de la información establece tres ámbitos para abordar las necesidades de los mayores. Su segundo ámbito de actuación hace referencia a (Comisión de las Comunidades Europeas, 2007):

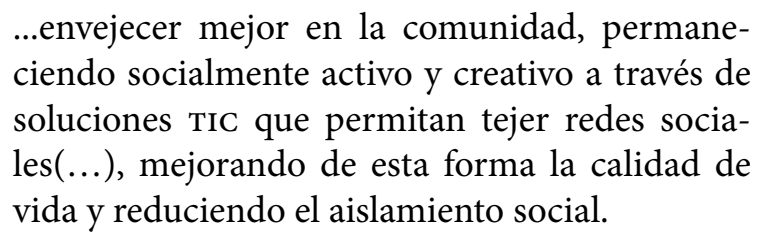

La parálisis cerebral (PC) es un grupo de trastornos permanentes del desarrollo, del movimiento y de la postura, que causan limitaciones en la actividad y que son atribuidos a alteraciones no progresivas ocurridas en el desarrollo cerebral del feto o del lactante. Los trastornos motores de la PC están a menudo acompañados por alteraciones de la sensación, percepción, cognición, comunicación y conducta, por epilepsia y por problemas músculoesqueléticos secundarios (Rosembaun, 2007).

La esperanza de vida se ha incrementando en las personas con PC. Se ha comprobado que el deterioro físico y psíquico que se empieza a constatar en cualquier persona mayor, en las personas con PC podemos hablar de proceso de envejecimiento a partir de 45 años, incluso antes.

Alguna de las características de las personas con PC en proceso de envejecimiento son las dificultades para las relaciones sociales, la falta de oportunidades para la interacción, la poca motivación y, además, un bajo nivel de tecnificación. La llamada brecha digital es más evidente en este colectivo, pues la desigualdad de oportunidades para el ac- ceso a la información, la comunicación y la educación ha de romper la doble barrera: ser mayor y tener discapacidad.

Dentro del Centro de Atención Integral Fuentecillas, en la Asociación de Personas con PC de Burgos y provincia (APACE), se encuentra el centro de día que presta servicio a personas a partir de 21 años. El centro está organizado en tres aulas específicas, siguiendo criterios de edad, necesidades de apoyo e intereses comunes. Una de ellas atiende a personas en proceso de envejecimiento.

Basándonos en que los mayores pueden aprender a través de programas y medios que tengan en cuenta las características personales y las circunstancias del sector (Montero, 2000), se planteó fomentar las relaciones sociales y generar nuevas formas de expresión utilizando las tecnologías de la información y la comunicación (TIC).

\section{Metodología}

Utilizando una metodología cualitativa basada en las entrevistas personales y la observación participante, se pudo detectar la presencia de dificultades en la comunicación tanto a nivel funcional como por falta de habilidades sociales, así como una escasez de motivación ante cualquier actividad. Las características concretas del aula de mayores nos llevaron a buscar herramientas que permitieran dar respuesta a estas dificultades. Por ello se creó un taller de introducción y manejo de las TIC.

La intervención docente se centró en personalizar la metodología partiendo del conocimiento de las capacidades de cada uno de los usuarios, adecuando la tecnología a cada persona en función de sus características físicas, personales y estilos de aprendizaje.

El taller se desarrolló a lo largo de un año, 2 días a la semana, con una duración de 2 horas por sesión. El grupo estaba formado por 12 participantes con edades comprendidas entre 45 y 62 años. En relación con el taller, se visitaron exposiciones sobre tecnología, se utilizó el apoyo de material audiovisual (videos, programas interactivos, etc.) y se aplicaron algunos aprendizajes en las $4^{\text {a }}$ Jornadas sobre PC en las que algunos participantes contaron sus experiencias con las TIC. Se trabajó de forma 


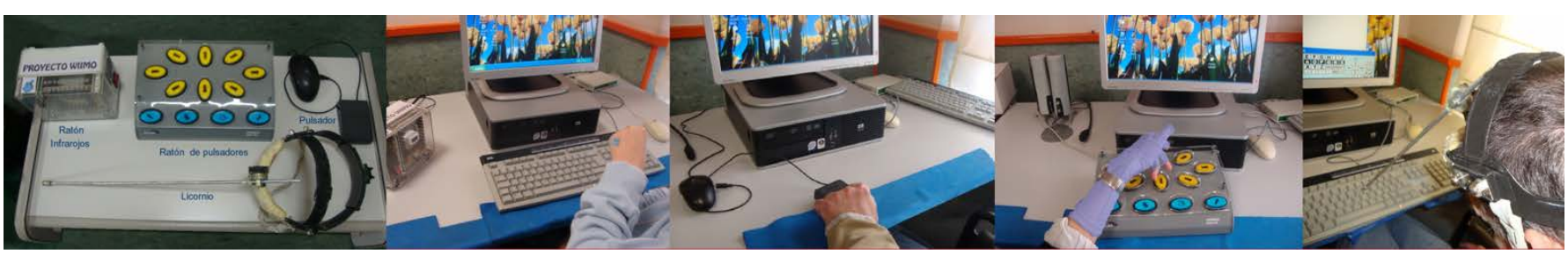

Figura 1. Productos de apoyo de acceso al ordenador

sincrónica los miedos e inseguridades, el rechazo, la vergüenza a equivocarse o mostrar su desconocimiento, intentando conectar con sus intereses personales, hablando de experiencias previas y haciendo sencilla la información para conseguir que la comprensión fuera más fácil.

Debido a las manifestaciones clínicas propias de la PC (alteración del tono, restricciones motoras, dificultad de movimiento selectivo, etc.) fue necesario dar solución a los problemas de acceso a los equipos informáticos. Se habilitaron las condiciones de acceso adecuadas para que cada una de las personas pudiera manejar los equipos. Se dispuso de punteros, ratones adaptados, pantallas táctiles, teclados de pantalla con posibilidad de ser configurados de forma personalizada, etc. (Figura 1).

De forma global, la alfabetización informática se llevó a cabo enseñando el manejo del entorno Windows, escribiendo textos, haciendo hincapié en la oportunidad de expresar emociones y sentimientos, buscando en Internet, comunicando y realizando transacciones de información, conocimientos, imágenes, etc., aprendiendo a compartir ideas y experiencias, participando en redes sociales y manejando el correo electrónico. Según cada uno de los participantes, y en función de intereses y capacidades, se trataron contenidos diferentes.

\section{Resultados y discusión}

Todos los participantes se mostraron satisfechos con sus logros en el taller. El 70 \% demandan a diario su utilización como medio de comunicación, así como para mantener las relaciones sociales creadas y provocar otras nuevas.

De acuerdo con la Comisión de las Comunidades Europeas y teniendo presentes las aportaciones del Libro Blanco sobre Envejecimiento Activo (2011), hemos intentado superar obstáculos y aprovechar oportunidades:

- Se comprobó que las personas con PC mayores se encuentran en situación de desventaja ante las TIC, ya sea por su situación personal, por la complejidad de la tecnología o porque los productos y servicios no están suficientemente adaptados o no existen, lo cual genera frustración y dependencia. Para compensar esta situación, partimos de las capacidades de cada usuario y, con la información y servicios existentes en el mercado, adaptamos los productos utilizando la creatividad y la imaginación.

- Las actitudes personales respecto a las TiC, los elevados costes y el desconocimiento de experiencias prácticas hacen que no se apliquen apoyos o adaptaciones específicas. Queremos difundir nuestra propuesta, desde el diseño para todos, y así romper estereotipos y facilitar la comercialización, no solo para nuestro colectivo, sino para toda la población.

- Las soluciones tecnológicas destinadas a los usuarios finales a menudo exigen la combinación o interconexión de diferentes servicios, herramientas y estrategias que desbordan la capacidad de las personas con PC. Siguen siendo muy necesarios los apoyos externos para el acceso.

- Carecen de un acceso básico a las redes de la comunicación. No disponen de equipos ni de conexión regular a Internet. Este menor acceso suele deberse a la falta de: motivación, medios económicos, competencias digitales y formación adecuada. Con esta experiencia, pretendemos conseguir un alto grado de visibilidad y sensibilización, buscar soluciones e incentivar su uso y aplicación. Dar a conocer programas de formación que incluyan la accesibilidad de los sitios web, la accesibilidad de las herra- 
mientas y servicios TIC convencionales y el diseño universal.

- Se ha encontrado en las nuevas tecnologías una herramienta útil para comunicarse con amigos y seres queridos a muy bajo coste, a través del correo electrónico, la mensajería instantánea y las redes sociales. Internet les permite participar en comunidades virtuales, con nuevos amigos, bien lejanos o cercanos que, por problemas de movilidad, no se pueden ver, favorece la amistad, la comunicación y el mantenimiento del entramado social.

- El empleo de las Tic facilita romper el estereotipo de la persona mayor anticuada y aporta recursos para una mejora de la calidad en su entorno relacional y familiar. Así, disponen de más y mejores oportunidades para la relación intergeneracional y para mantener relaciones desde roles más valorados.

\section{Conclusiones}

Se ha intentado desarrollar e implantar herramientas y servicios TIC de fácil utilización, integrando las necesidades de los usuarios según sus capacidades y apoyando otros ámbitos estratégicos ante los retos que plantea el envejecimiento, aumentando la sensibilización, construyendo estrategias comunes e individualizando, eliminado obstáculos técnicos y fomentando la adopción de las tecnologías. Se debe coordinar y reforzar el trabajo existente, abordar las necesidades de los usuarios para que permanezcan socialmente activos a través de soluciones TIC que permitan tejer redes sociales, mejorando de esta forma la calidad de vida y reduciendo el aislamiento social.

Sin embargo, queda mucho camino para lograr que los mayores con PC puedan participar plenamente, en el momento y el lugar que deseen, en la sociedad y permanecer activos como ciudadanos capacitados. Nuestro deseo es seguir explorando el prometedor potencial de las TIC asociadas al proceso de envejecimiento de las personas con parálisis cerebral como líneas futuras de investigación.

\section{Referencias}

Comisión de las Comunidades Europeas, C. (2007). Envejecer mejor en la sociedad de la información. Una iniciativa i2010. Plan de acción sobre Tecnologías de la Información y la Comunicación y envejecimiento. Bruselas.

Instituto de mayores y Servicios sociales (2011). Libro Blanco de Envejecimiento Activo. Madrid: Ministerio de Sanidad, Política Social e Igualdad, Secretaria General de Política social y consumo e Instituto de mayores y Servicios sociales. Disponible en: http://www.imserso.es/imserso_01/envejecimiento_activo/libro_blanco/index.htm. [Acceso: 25/2/2012].

Montero, I. (2000). Nuevas perspectivas en el aprendizaje de los mayores. En M. V. Alcalá, El aprendizaje de los mayores ante los retos del nuevo milenio. Madrid: Dykinson.

Ochoa, E. (2010). Nuevos avances en la accesibilidad Tic. En P. H. Arnaiz, 25 años de integración escolar en España: Tecnología e inclusión en el ámbito educativo, laboral y comunitario. Murcia: Consejería de Educación, Formación y Empleo.

Rosembaun, P. P. (2007). A report: the definition and classification of cerebral palsy. Development Medicine Child Neurology , 49 (supl 2), 8-14. 